METALLURGY AND FOUNDRY ENGINEERING - Vol. 37, 2011, No. 1

\author{
Anna Derda*, Maciej Nadolski**, Karolina Muskała*
}

\title{
LIFECASTING IN ARTISTIC CASTING
}

\section{INTRODUCTION}

From the dawn of history artistic castings offer man the opportunity to preserve a shape of the transient, especially of men who deserved commemoration for various reasons. The technique which enables to avail directly of a living body as a primary model is the lifecasting (LC) method. Lifecasting is a technology of three-dimensional portraying of a living object using casting materials and technologies. This way of casting is not very popular in Poland yet, and even the term 'lifecasting' has no Polish equivalent so far.

The beginning of lifecasting techniques dates back to antiquity, when the so-called death masks were taken for commemorating faces of the deceased. Those masks were made of plaster, wax, or indirectly in metal. They took an important place as religious objects in funeral rites of ancient Egypt and Rome. Thanks to such masks we are acquainted with the exact appearance of such historical persons as William Shakespeare, Napoleon I Bonaparte, Isaac Newton, Joseph Piłsudski and many more [1-3]. At the beginning of the XX century the death masks lost their significance due to the development of photography, however the technique itself gave birth to lifecasting - an alternative to sculpture, but quicker and cheaper way of three-dimensional portraying [1]. Lifecasting tries to get a separate place in artistic casting and sculpture, and it is a challenge to many artists searching for new means of expression and quick methods of work.

At present lifecasting is quite popular in United States and in some of European countries, e.g. Great Britain, Germany, and Sweden [4]. The reason for a constant spreading of this technology is the common availability of new, time-saving and easy-to-handle

* M.Sc., ** Ph.D.: Department of Foundry, Technical University of Czestochowa, Czestochowa, Poland; e-mail: a.derda@wip.pcz.pl 
materials (alginates, synthetic plastics, elastomers) which remarkably surpass traditional materials with regard to their physical properties. For many years plaster slurries along with low-melting metals and alloys have played the main part as materials for preparing patterns and moulds. Nowadays there exist a considerably wider variety of materials which can be applied for this purpose [6-10]. Several features should be taken into account while choosing a material for preparing the so-called 'negative' of human body, e.g. material consistency, elasticity and ability to reproduce surface details. It is also important that the material intended for a matrix should not involve allergic reactions, should have a pleasant smell, short setting time and should not exhibit volume changes during its setting. These requirements induce the use of three main types of materials for the LC technology. They are alginate, silicone, and plaster mixtures. Lifecasting is most frequently applied for replicating human faces, hands, feet, and torsos. This method is also used for copying plants, along with the flowers. Castings produced in this way are characterised with a high level of realism in reproducing of the model surface, often replicating such tiny details as skin pores or ridges. It should be remembered, however, that - in contrast to the casting from an inanimate subject - lifecasting can create some risk to the model. The possible dangers are e.g. allergic reactions involved by the material of a mould, difficulties with breathing freely during the process of face copying, or even fainting [5, 9].

Production of a metal casting according to the lifecasting method requires for additional auxiliary methods. Lifecasting is successfully supported by the methods of precision and artistic casting, in particular by investment casting technology. Investment casting allows for achieving castings of intricate shapes, large dimensional accuracy, and small surface roughness [11].

\section{THE SCOPE OF INVESTIGATIONS}

The work has been aimed to develop a lifecasting technology for replicating human face and head with use of alginate impression materials. Some presumptions has been taken prior to the experimental work. A change of the recipe for the impression material has been recognized as sensible with regard to the lifecasting technology, so that the liquid consistency of the mixture can be achieved for the purpose of both precise reproducing without pushing (customary in dentistry) and prolonging the working time. It has been assumed that the elastic matrices reproducing the human body parts would be made of the 'Tropicalgin' prosthetic-stomatologic material. A detailed description of the LC process is presented here for the examples of replicating a woman's face and a man's head. These experiments on living persons have been preceded by practising on simpler elements of human body, such as hands and feet (Figs. 1-2). The obtained trial castings have been made in the same way as it is further described for producing a casting of woman's face. 

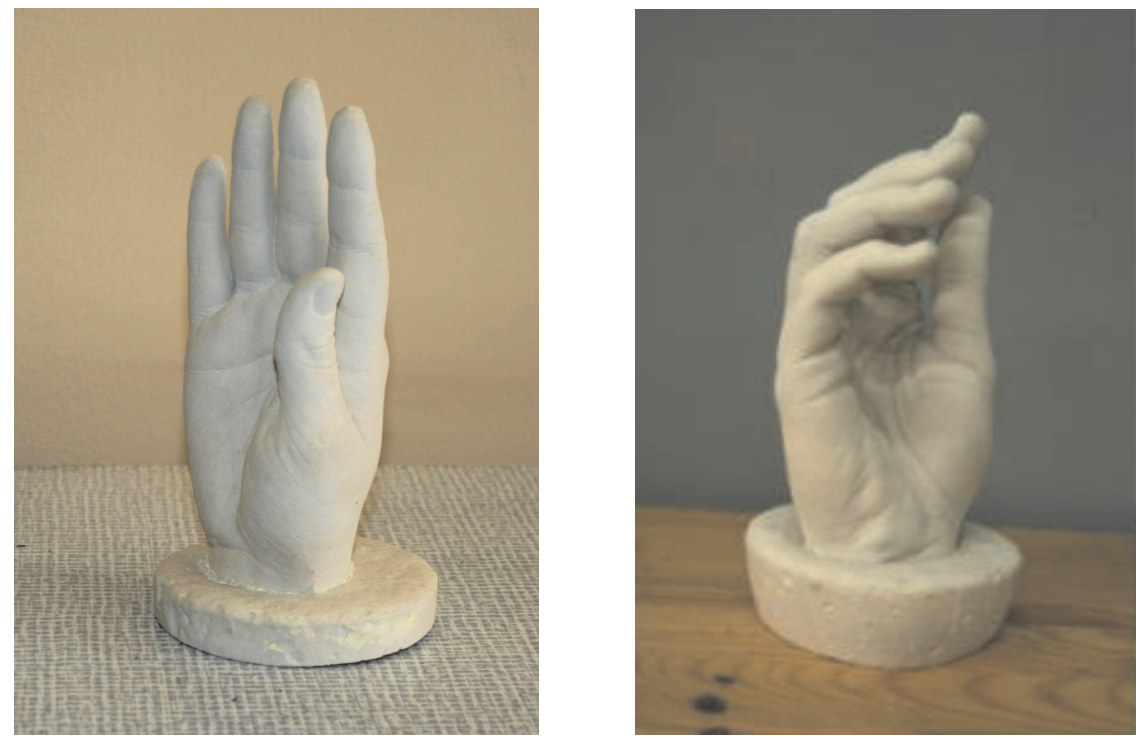

Fig. 1. Plaster casting of hands

a)

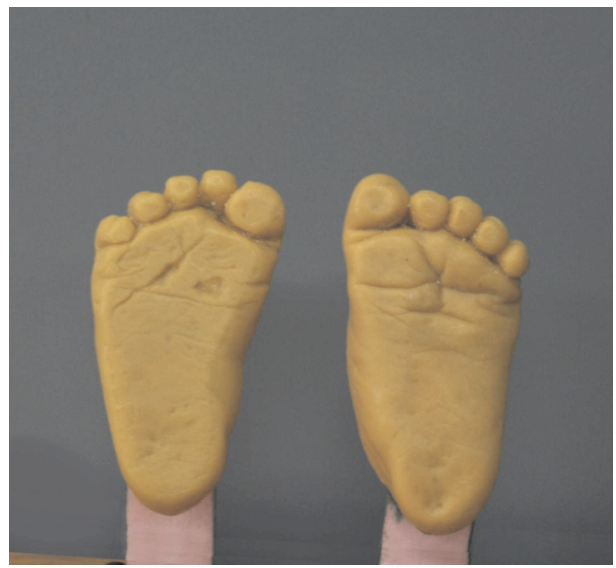

b)

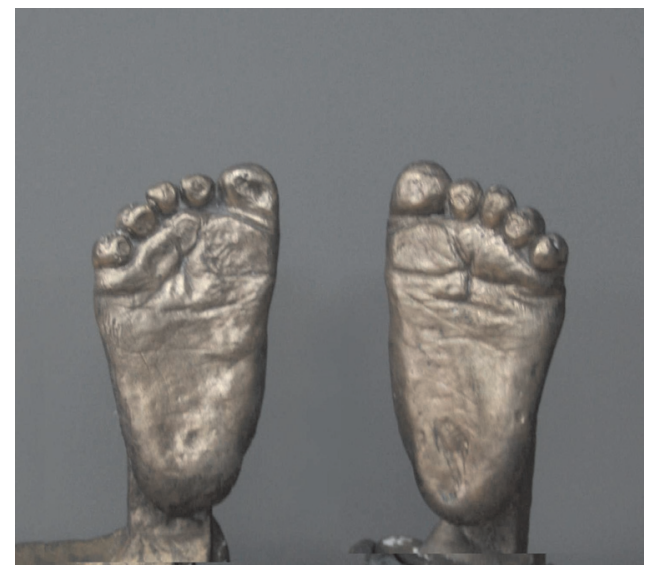

Fig. 2. Baby feet replicated by Lifecasting: a) wax patterns; b) bronze castings

\subsection{Modification of mixture for use in the lifecasting method}

Chromatic alginate impression material commonly available under the 'Tropicalgin' trademark and used in dentistic prosthetic for making impressions in the process of producing stable and movable prostheses has been used for making the model replicas. The material is safe for man and exhibits short setting time which is determined by the manufacturer as equal to $3 \mathrm{~min} 35 \mathrm{sec}$. 
The selection of a suitable proportion of alginate mixture components has consisted in determination of the technological properties of mixture containing variable amounts of water and reinforcement in the form of polypropylene fibre. The quantities of mixture components used in experiments are presented in Table 1.

The mixtures prepared according to the given recipe have been examined with respect to their fluidity and setting time. The determination of fluidity has consisted in putting a mixture into a cylinder of $50 \mathrm{~mm}$ height and $46 \mathrm{~mm}$ diameter placed in the middle of a plate with marked concentric circles of diameters ranging from 100 to $240 \mathrm{~mm}$ with intervals of $10 \mathrm{~mm}$, constituting a kind of measurement graduation. Then the cylinder has been risen up with a constant velocity, and the diameter of mixture spreading has been determined. The Vicat apparatus has been used for determining the setting time of the examined mixture with the same method as the one applied for moulding materials. The obtained results are presented in Table 1.

Table 1. Content of individual components of alginate mixtures and the examination results concerning the fluidity and setting times of the prepared mixtures

\begin{tabular}{|c|c|c|c|c|c|c|}
\hline \multirow{2}{*}{$\begin{array}{c}\text { No. } \\
\text { of measurement }\end{array}$} & $\begin{array}{l}\text { Quantity } \\
\text { of water }\end{array}$ & $\begin{array}{l}\text { Quantity } \\
\text { of fibre }\end{array}$ & $\begin{array}{l}\text { Alginate } \\
\text { mass }\end{array}$ & Fluidity & $\begin{array}{c}\text { Setting } \\
\text { time }\end{array}$ & \multirow[t]{2}{*}{ Notes } \\
\hline & $\mathrm{cm}^{3}$ & $\mathrm{~g}$ & $\mathrm{~g}$ & $\mathrm{~mm}$ & $\mathrm{~s}$ & \\
\hline 1 & 80 & 0.5 & 10 & 145 & 170 & Thick mixture \\
\hline 2 & 80 & 0 & 10 & 155 & 160 & Thick mixture \\
\hline 3 & 110 & 0.5 & 10 & 170 & 190 & \multirow{2}{*}{$\begin{array}{l}\text { Highly-fluid } \\
\text { mixture of long } \\
\text { setting time }\end{array}$} \\
\hline 4 & 110 & 0 & 10 & 180 & 200 & \\
\hline 5 & 95 & 0.25 & 10 & 170 & 120 & \multirow{3}{*}{$\begin{array}{c}\text { Fluid mixture } \\
\text { of short setting time }\end{array}$} \\
\hline 6 & 95 & 0.25 & 10 & 170 & 135 & \\
\hline 7 & 95 & 0.25 & 10 & 180 & 125 & \\
\hline
\end{tabular}

The results gathered in Table 1 have been a basis for statistical analysis aimed to find a statistically significant mathematic model describing the fluidity of a mixture $(y)$. It has been determined as follows:

$$
y=16.75+1.25 x_{1}
$$

where $x_{1}$ stands for water quantity measured in millilitres.

Taking into account the fact that the Equation (1) is valid only for the analysed content range, it should be noticed that the fluidity of the examined mixture depends only on the content of water, and is not influenced by the content of polypropylene fibre within the examined range of changes. 


\subsection{Reproduction of a face according to the lifecasting technology}

The first stage of the process has consisted in making a mould using the alginate powder in the form of 'Tropicalgin' impression material and distilled water of temperature $11^{\circ} \mathrm{C}$ mixed in proportion 1:7, while the proportion used in dentistry is 1:2. Applying water of lower temperature than the recommended by the manufacturer has allowed for additional extending of the working time. The mixing, the working, and the setting times have been controlled by means of dyes added to the alginate powder (comp. Fig. 3).

a)

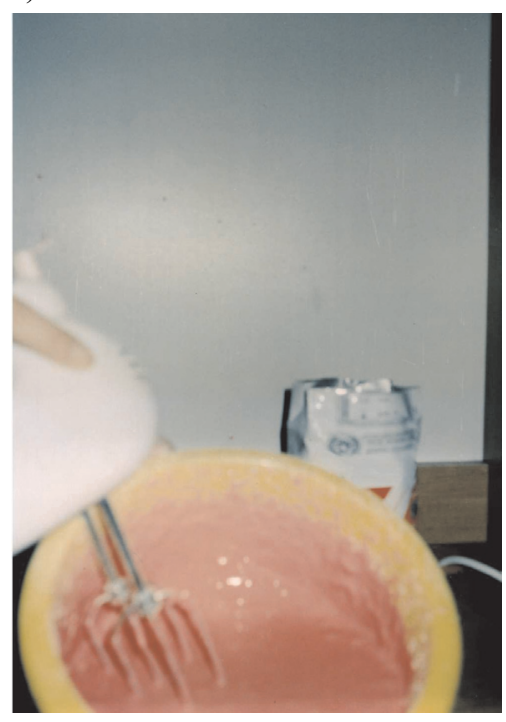

b)

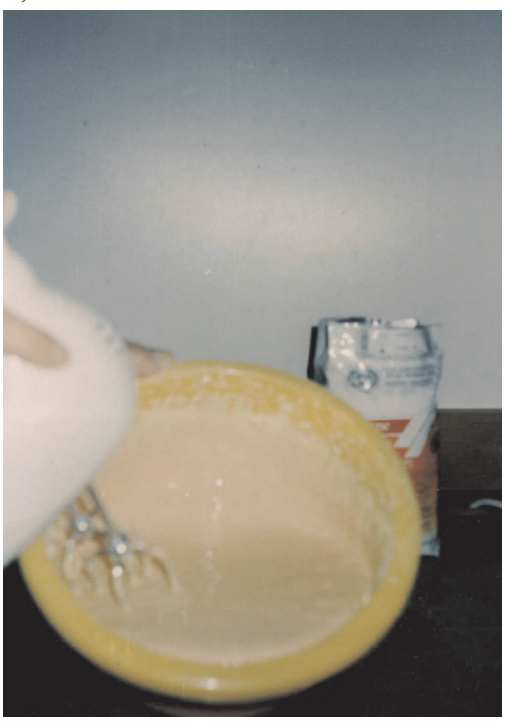

Fig. 3. Preparing alginate mixture, mixing time: a) $2 \mathrm{~min}$; b) $2 \mathrm{~min} 45 \mathrm{sec}$

The copying process has been started with applying a thin layer of cream creating a protective barrier for model's skin and eyebrows. After covering the whole face with an alginate layer (Fig. 4) and after setting of the material, the alginate layer have been overlaid with cotton gauze with plaster in order to stiffening the matrix number 1 and to minimize the possible tearing or distortion of the elastic material during further operations (Fig. 5). During the copying process the model has breathed through a pipe in her mouth. After the mould has been made and taken off (Fig. 6), it has been filled with plaster to achieve the replica. The plaster casting obtained from the mould is shown in Figure 7.

Using the pipe for breathing (see Fig. 4) has caused the defect of replica mouth (Fig. 7) and the necessity of local correction of the replica No. 1. It has been decided to leave this first replica untouched and to make the subsequent plaster replica (No. 2) for reconstruction and refining of details. To do this, the No. 2 matrix has been prepared of GUMOSIL WW soft silicone rubber of increased strength using the 60R catalyst. Silicone matrix has been 
strengthened with a casing of plaster for pattern making. The corrections have been made using stomatologic plaster of the same hardness, but of different colour to make them easy to distinguish.

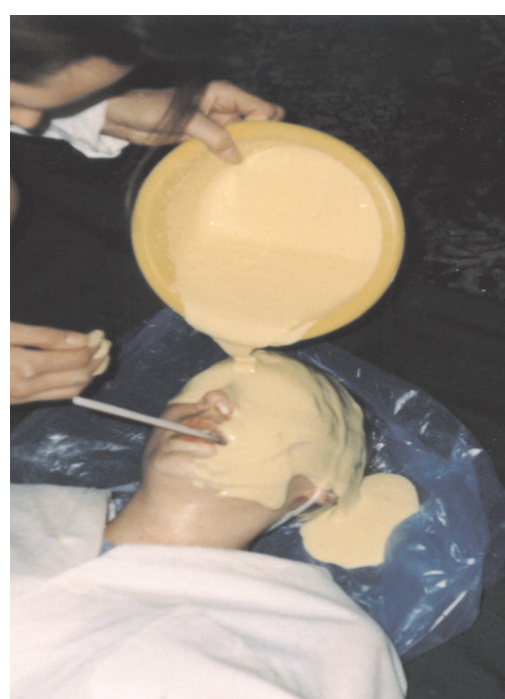

Fig. 4. Applying the alginate mixture on the greased face

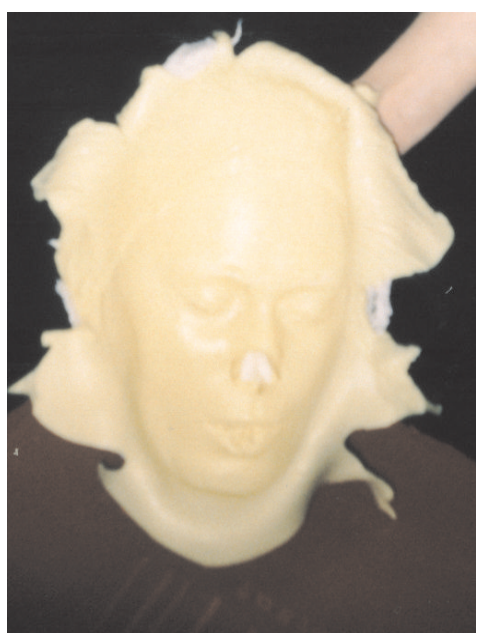

Fig. 6. The alginate matrix No. 1

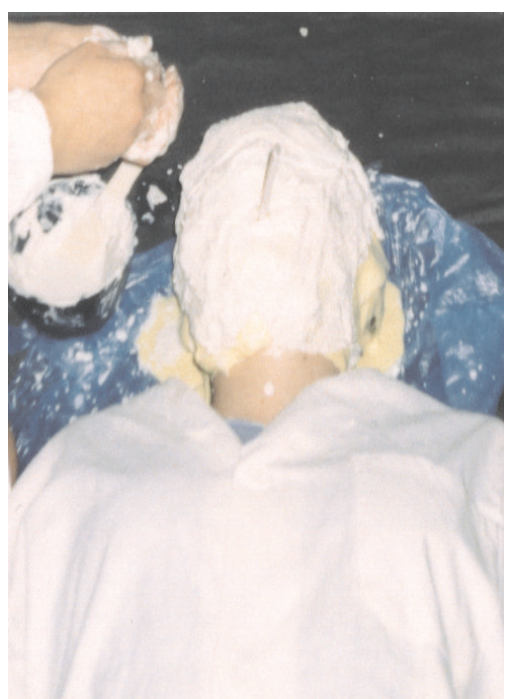

Fig. 5. Strengthening of alginate matrix using cotton gauze with plaster

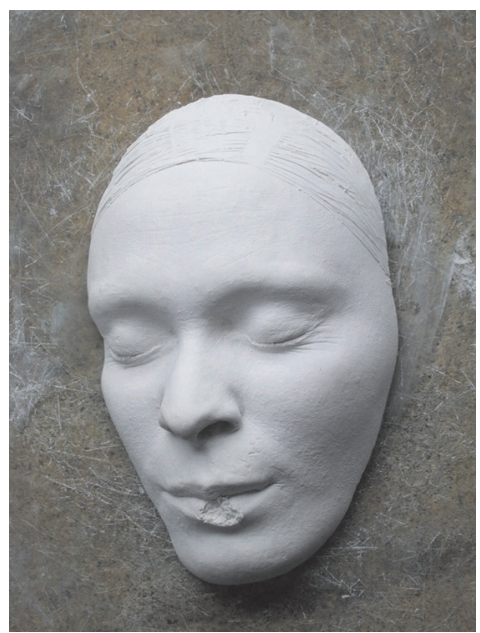

Fig. 7. The plaster casting obtained from the alginate matrix

Lifecasting is a method by which one cannot obtain a metal casting directly, therefore it has been necessary to employ a supplementary method, namely the investment casting. 
Further work consisted in preparing the rigid-elastic matrix (No. 3 mould) from the plaster replica No. 2. This third mould has been made of the above mentioned rubber and catalyst. After the curing process had been finished, the mould has been strengthened with a plaster casing. The No. 3 mould has been used for production of wax replicas made of the commercially available 'CASTALDO' wax (Fig. 8).

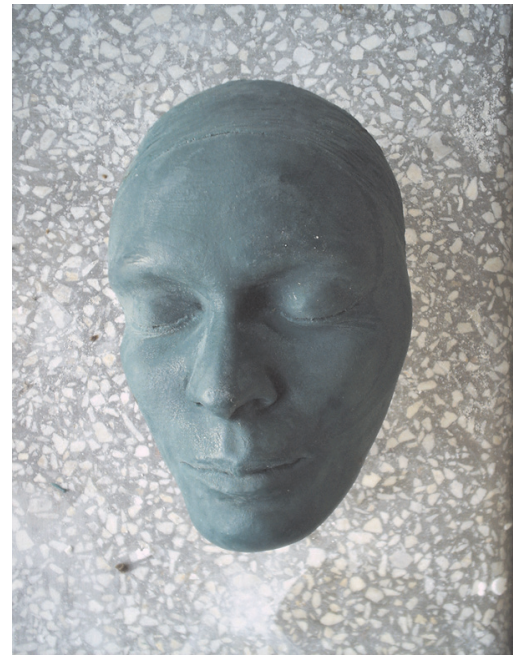

Fig. 8. The prepared wax pattern

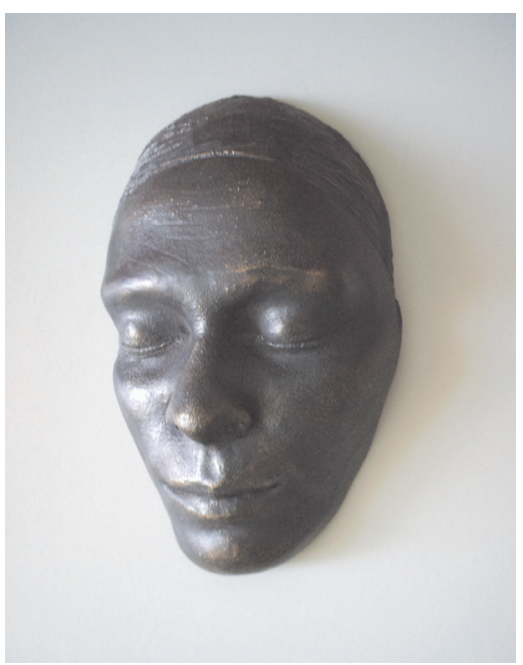

Fig. 9. The bronze casting obtained by the lifecasting method

The material used for building a full, block ceramic mould for metal casting has been plaster for pattern making, silica sand, and water. Mixed components have been degassed in the HYPERVACUUM 50 vacuum chamber made by Mario di Maio Company. Then the mixture has been poured onto the previously prepared wax pattern set and left for 24 hours to achieve the full setting of the material. The wax pattern has been melted in a laboratory drying oven at the temperature of $200{ }^{\circ} \mathrm{C}$ for 8 hours. Subsequently the mould has been placed in the furnace for heat treatment and has been heated at first at the temperature of $550{ }^{\circ} \mathrm{C}$ for 4 hours, then at $740{ }^{\circ} \mathrm{C}$ for another 4 hours. After finishing the burnout process, the mould has been cooled with the furnace down to $380^{\circ} \mathrm{C}$ and transferred to the pouring stand.

The CuSn5Zn5Pb5 alloy has been used for making the final casting of the face. After removing the elements of gating system and fettling, there has been applied the coloured patina endowing the final appearance shown in Figure 9.

\subsection{Lifecasting - man's head}

Casting of the head of a living man has been performed in a way similar to the developed process of reproducing the human face, with some differences described beneath.

Applying of the divided alginate matrix, consisting of four parts reproducing the hair arrangement, the face, and both ears, has been reckoned to be reasonable. Determining of 
parting lines positions has been here a matter of importance. The material used for preparation of the elastic matrix has been the 'Tropicalgin' mixture of composition given in Table 1 (the measurement No. 1).

First the back part of the matrix has been made, then the face has been copied, starting the alginate application from the forehead down to the neck. The last stage comprised the preparation of the matrices of ears. After stiffening the matrix with a plaster casing, it has been taken off the model. Then its parts have been put together again in order to cast a plaster replica. Figure 10 shows the process of removing the alginate mould from the plaster replica. The completed replica after some cleaning in presented in Figure 11.

a)

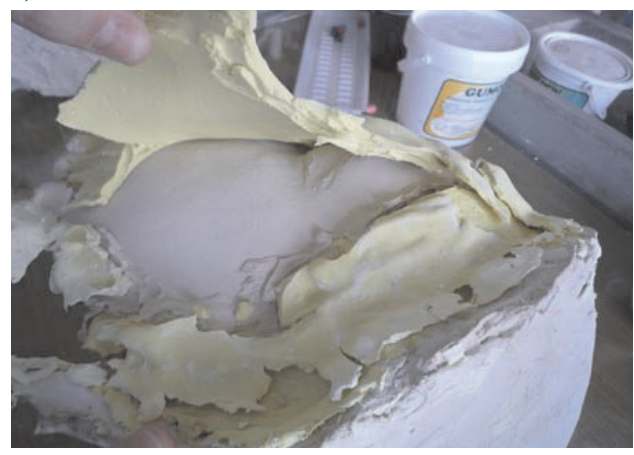

b)

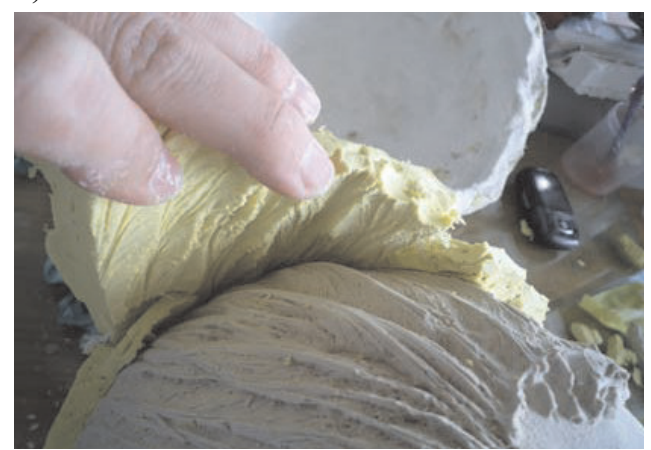

Fig. 10. Removing the mould from the plaster replica: a) from the face; b) from the hair

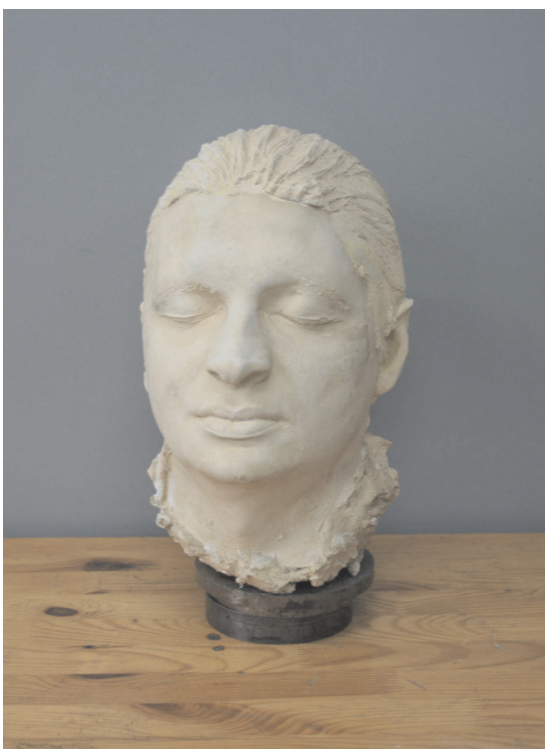

Fig. 11. The replica of the model

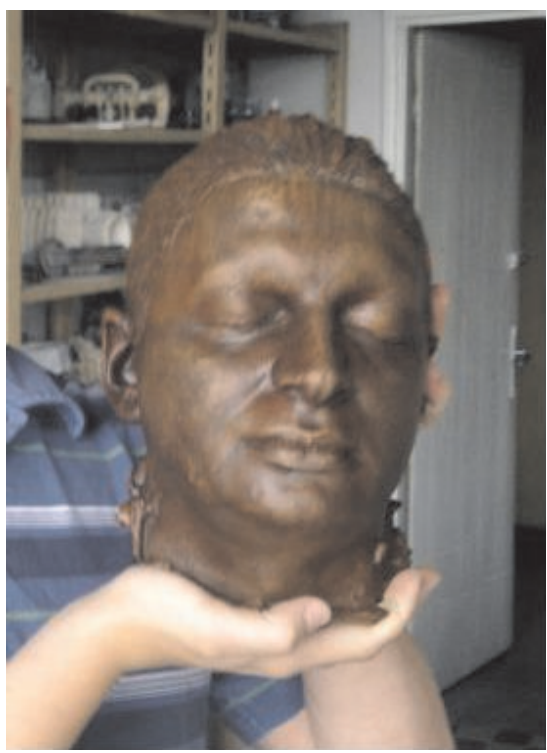

Fig. 12. The bronze casting of man's head obtained by LC method 
The subsequent stage has comprised the advance from replicating process to the investment casting. The same procedure has been followed as the above described one. The final effect of the work i.e. the bronze casting of CuSn5Zn5Pb5 tin-zinc-lead bronze is depicted in Figure 12.

\section{SUMMARY}

It has been found in the course of investigation work that, for a constant quantity of alginate, the fluidity of the material depends on the quantity of water, and is not influenced by the quantity of polypropylene fibre added to the mixture (Eq. 1). It has been noticed during the matrix removing that the increased quantity of water influences not only the material fluidity, but also its mechanical properties, probably diminishing them. This results in a risk of damaging the matrix during its removing from the model. The obtained results (Table 1) allow for supposing that the quantity of water does not matter for the setting time of alginate mixture.

The developed lifecasting technology is absolutely safe with regard to the model health, and the applied modern materials for copying do not contain either toxic or allergic, or skin-irritating substances. Applying of the investment casting technology for realisation of the final bronze casting has allowed for the excellent reproduction and achieving the high surface quality.

It has been confirmed that making a copy of human head is the most difficult process within the scope of lifecasting technology. It is both time- and material-consuming technique, which requires for an enormous patience and precision of the matrix makers. Moreover, carrying out of the copying process demands the practical knowledge with concern both to the applied materials and to the replicating procedure itself. It should be stressed that the developed technology allows for excellent reproduction of the copied body parts, retaining - despite the multiple copying - even tiny details, such as skin ridges.

Lifecasting is an interesting alternative for artistic casting workshops and sculpture studios, allowing for quick realisations of orders, particularly of three-dimensional portraits. It is even more interesting due to the fact that the precise portraying does not demand the outstanding sculptural talents from the author.

\section{REFERENCES}

[1] McCormick E.: Death Masks Give Birth to Lifecasting, Art Casting Journal [online], August 2001, Access protocol: www.artmolds.com [04.11.2010]

[2] Kolbe G., Green M.M.: Undying Faces: A Collection of Death Masks, Kessinger Publishing LLC, 2003

[3] Virtual Museum of Death Mask, Access protocol: www.deathmask.kiev.ua [04.11.2010]

[4] Association of Lifecasters International, Access protocol: www.lifecasting.org [05.11.2010]

[5] Debreceni T: Special Makeup Effects for Stage and Screen, Elsevier Science, 2008

[6] Rosier P.: Le Moulage, Editeur: Bordas, 1998 
[7] Combe E.: Notes on dental materials, Churchill Livingstone, Edinburgh, 1992

[8] Kordiasz P., Wolanek Z.: Practical dental materials technology, Państwowy Zakład Wydawnictw Lekarskich, Warszawa, 1976

[9] McCormick E.: Lifecasting . . Sometimes It Is For The Faint of Heart, Art Casting Journal, 2001, Access protocol: www.artmolds.com [06.11.2010]

[10] McCormick E.: Alginate - Lifecasters' Gold, Art Casting Journal, 2001, Access protocol: www.artmolds.com [06.11.2010]

[11] Wierzbicka B., Soiński M.: Casting technology, Skrypt Politechniki Częstochowskiej, Częstochowa, 1996

Received

February 2011 